\title{
Solving the mystery of H7N9 by crystal balls
}

\author{
Cell Research (2013) 23:1335-1336. doi:10.1038/cr.2013.150; published online 12 November 2013
}

\begin{abstract}
How the novel influenza H7N9 virus crossed species barrier from avian to human is intriguing. Extrapolation from previous studies on H5N1 can be misleading as illustrated by crystallographic studies on the $\mathrm{H} 7$ hemagglutinin with G226L substitution; crystal structure of the neuraminidase N9 showed that R294K substitution interferes with binding to sialic acid or antiviral drugs and reduces viral fitness.

Influenza A virus is subtyped by its
\end{abstract} two major surface proteins, the hemagglutinin (H1 to H18) that attaches the virus to host sialic acid receptor and the neuraminidase $(\mathrm{N} 1$ to $\mathrm{N} 11, \mathrm{~N} 10$ lacks enzymatic function) that releases the progeny virus from infected cells by hydrolyzing the sialic acid receptor. Historically, seasonal and pandemic influenza due to human-to-human transmission are caused by subtypes of combinations of $\mathrm{H} 1, \mathrm{H} 2$ and $\mathrm{H} 3$ with N1 and N2. Since 1997, outbreaks or sporadic human infections due to avian-to-human transmission of avian influenza A virus subtypes $\mathrm{H} 5 \mathrm{~N} 1$, H9N2, H7N7, H7N2, H7N3, H10N7 and $\mathrm{H} 6 \mathrm{~N} 1$ have been reported. Except for the H5N1 virus that killed nearly $60 \%$ of all reported cases, most of these human cases were self-limiting mild acute upper respiratory tract infections and conjunctivitis. However, in 2013, the H7N9 virus emerged in China, which has affected 135 patients with 45 deaths. This number of H7N9 patients is over three times the total number of H5N1 patients in China within the last ten years [1]. Phylogenetic analysis of the human and avian virus isolates from epidemiologically-linked live poultry market suggested that patients acquired the H7N9 virus from contacts with live poultry [2]. Furthermore, genetic analysis of these viral isolates showed evidence of mammalian adaptation. Two important questions have to be answered urgently. How does this novel virus jump from poultry to human and what drug treatment can be used to treat this disease with such a high mortality of $33 \%$.

A group of scientists from the Chinese Academy of Sciences/China CDC led by Gao GF gave these important answers by a combination of conventional virology, reverse genetics and crystallography within a period of just six months. Gene sequencing of the human Shanghai/H7N9 virus showed that it has a Gln at amino acid position 226 of H7 (H3 numbering) whereas the Anhui/H7N9 virus has a Leu at the same position. Extrapolations of data from the $\mathrm{H} 5$ of $\mathrm{H} 5 \mathrm{~N} 1$ virus suggested that the substitution of Leu for Gln will increase the virus binding to human receptor with $\alpha$-2,6-linked galactose and reduce the virus binding to avian receptor with $\alpha$-2,3-linked galactose. While the Shanghai/H7 indeed preferentially binds avian receptor, the Anhui/ H7 binds both avian and human receptors. The group was not satisfied with this finding and went on to construct an Anhui/H7 mutant with a Leu-to-Gln substitution, which surprisingly still binds well to both avian and human receptors [3]. This throws into doubt the importance of the substitution in switching the specificity of host receptor from avian to human. Indeed, the elegantly done crystallography of the Anhui/H7 in complex with the human receptor analogue showed that the four hydrophobic amino acid residues around the receptor-binding pocket (Ala138, Val186, Pro221, Leu226) stabilized the cis conformation of the human receptor (Figure 1A). Even in the presence of the substitution by Gln226, the other three hydrophobic residues can maintain this binding conformation. This finding has practical significance in explaining why the novel H7N9 virus can jump from poultry to human but has limited human-to-human transmissibility up to this stage. The retention of the relatively high affinity for avian receptors by the H7N9 virus may help to trap the virus in the mucus of the human upper respiratory mucosa that secretes abundant mucin molecules rich in sialic acid receptor with $\alpha-2,3$ linked galactose. It would be important to monitor further amino acid changes around the receptor-binding pocket of the hemagglutinin with the resurgence of this H7N9 epidemic in the coming winter.

After solving the H7 mystery, the group went on to solve the riddle of another important surface protein with enzymatic function, the N9, which historically the first time jumps from avian to human. This surface neuraminidase is the target of the commonly used antiviral agents such as oseltamivir, peramivir and zanamivir. Gene sequencing of the Shanghai/N9 showed an Arg-to-Lys substitution at amino acid position 294 (equivalent to 292 in N2 numbering) that is also known to confer oseltamvir resistance in $\mathrm{H} 3 \mathrm{~N} 2$ and $\mathrm{H} 11 \mathrm{~N} 9$, while the Anhui/N9 has Arg at this position and is susceptible to neuramindase inhibitors. They used reverse genetics to generate a panel of H7N9 mutants and 
A

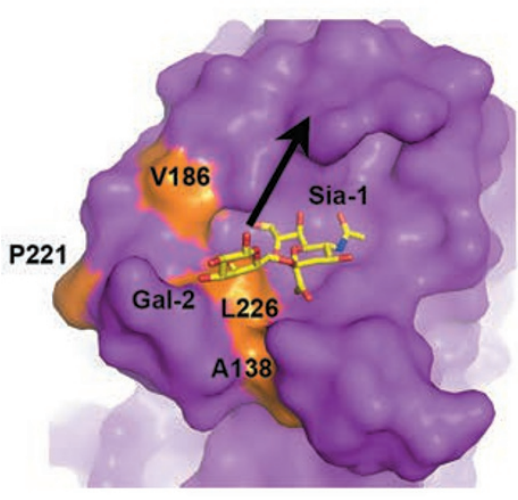

Anhui H7 L226Q mutant / human receptor

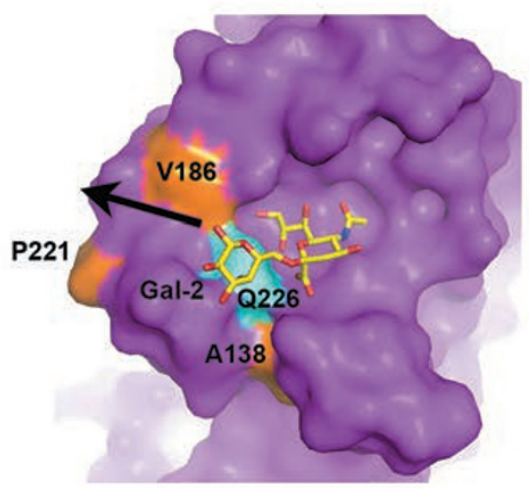

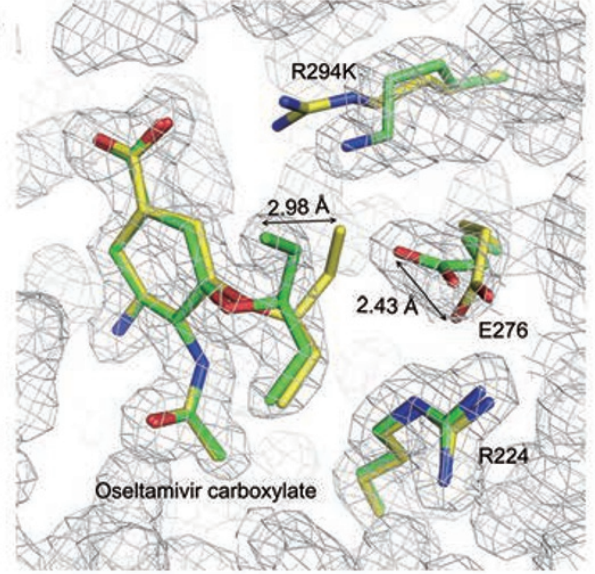

Figure 1 (A) Surface presentations of Anhui-H7 and Anhui-H7 L226Q mutant bound to human receptor analogues [3]. When Anhui$\mathrm{H} 7$ binds to the human receptor analogue, the Gal-2 ring (the second galactose moiety) is oriented face on, thus the remaining glycan rings could fold back to exit towards the right side of the receptor-binding site. When Anhui- $\mathrm{H} 7 \mathrm{~L} 226 \mathrm{Q}$ mutant binds to the human receptor analogue, the Gal-2 ring is oriented edge on, thus the remaining glycan rings would extend towards the left site of the receptor-binding site, which is consisted of residues P221 and V186. The residue A138 could also contribute some hydrophobic effect to compensate for the L226Q substitution. (B) The superimposed structures of the active sites of Anhui N9 (yellow) and Shanghai N9 (green) are shown with bound oseltamivir carboxylate colored similarly; relevant portions of electron density maps are also shown [4]. E276 in Shanghai N9 is unfavorable to accommodate the oseltamivir carboxylate pentyloxy group, thereby pushing the oseltamivir carboxylate hydrophobic group away from the active site $(2.98 \AA)$.

showed that the resistant N9 genotype is associated with extreme resistance to oseltamivir, decreased susceptibility to peramivir, laninamivir and zanamivir, decreased replication efficiency and neuraminidase activity [4]. R294 is one of the three key conserved active site arginines at the tri-arginyl cluster that is thought to play a key role in distorting the sialic acid pyranose ring from a chair to a boat conformation. This is the critical step for N9 to catalyze the hydrolysis of the terminal sialic acid from the receptor or its analogues. The group has rapidly generated the crystal structures of N9 in complex with neuraminidase inhibitors and showed that unlike arginine, lysine in the R294K mutant is out of range to form any typical hydrogen bonds with Y406 or E277 [4] (Figure 1B). Instead, lysine forms a salt bridge with E276 to adopt a conformation that interferes with the binding to oseltamivir carboxylate and its change from chair to boat conformation. These findings suggested that R294K mutants are less fit and likely to emerge mainly in patients who are under oseltamivir treatment. This also explained why R294 mutants were found mainly in patients already treated with oseltamivir and dead patients despite the treatment. While neuraminidase inhibitors should remain as the recommended drug treatment for influenza A H7N9, careful monitoring for the emergence of resistant mutants would be necessary. Though crystallography often gives an impression of definitive but very slow, these two studies have exemplified the importance and timely application of structural biology and biophysics in the fight against emerging infectious diseases.

\section{Kwok-Yung Yuen ${ }^{1}$}

${ }^{1}$ State Key Laboratory for Emerging Infectious Diseases, Department of Microbiology, The University of Hong Kong, Pokfulam Road, Pokfulam, Hong Kong SAR, China

Correspondence: Kwok-Yung Yuen

E-mail: kyyuen@hkucc.hku.hk

\section{References}

1 To KK, Chan JF, Chen H, et al. Lancet Infect Dis 2013; 13:809-821.

2 Chen Y, Liang W, Yang S, et al. Lancet 2013; 381:1916-1925.

3 Shi Y, Zhang W, Wang F, et al. Science 2013; 342:243-247.

4 Wu Y, Bi YH, Vavricka CJ, et al. Cell Res 2013; 23:1347-1355. 